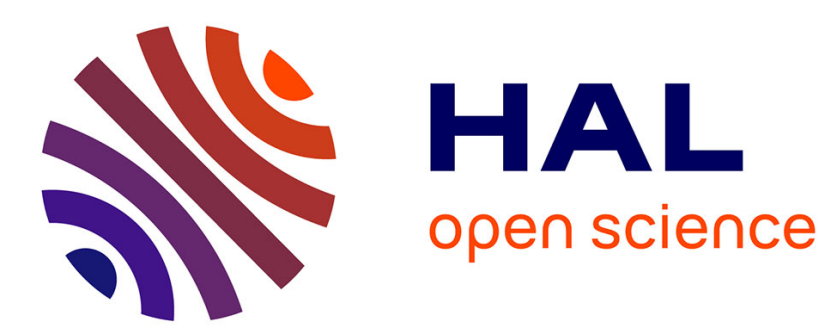

\title{
Les pratiques de recherche d'information informelles des jeunes sur internet
}

Karine Aillerie

\section{To cite this version:}

Karine Aillerie. Les pratiques de recherche d'information informelles des jeunes sur internet. 2008. sic_00344181

\author{
HAL Id: sic_00344181 \\ https://archivesic.ccsd.cnrs.fr/sic_00344181 \\ Preprint submitted on 3 Dec 2008
}

HAL is a multi-disciplinary open access archive for the deposit and dissemination of scientific research documents, whether they are published or not. The documents may come from teaching and research institutions in France or abroad, or from public or private research centers.
L'archive ouverte pluridisciplinaire HAL, est destinée au dépôt et à la diffusion de documents scientifiques de niveau recherche, publiés ou non, émanant des établissements d'enseignement et de recherche français ou étrangers, des laboratoires publics ou privés. 
Karine AILLERIE

Professeure-documentaliste certifiée

Doctorante karine.aillerie@ac-poitiers.fr

Adresse universitaire : LABSIC. Université Paris 13 UFR des Sciences de la communication 99, av. Jean-Baptiste Clément 93430 Villetaneuse

Titre : Les pratiques de recherche d'information informelles des jeunes sur internet

Résumé : Les grandes enquêtes décrivant les usages assidus et exclusifs d'internet par les adolescents privilégient la dimension communicationnelle et ludique de ces pratiques. En particulier depuis la parution de l'enquête internationale Médiappro, menée en France à l'initiative du CLEMI, l'accent est mis sur l'écart qui sépare d'une part, les habitudes domestiques, individuelles et quotidiennes, relevant de l' «informel», des usages scolaires et encadrés d'autre part. La question de cet écart reste posée en ce qui concerne les pratiques de recherche d'information réelles des jeunes internautes, encore insuffisamment appréhendées. Ce sont finalement les capacités mêmes qu'ils sont susceptibles de développer au gré de ces usages qui sont ici interpellées.

Mots-clés : pratiques d'information, jeunes, internet, informel, rapport au savoir, compétences informationnelles, savoirs informationnels 
Les pratiques de recherche d'information informelles des jeunes sur internet

La littérature liée au thème des jeunes et internet est vaste et hétérogène. Les enquêtes statistiques ou les réflexions généralistes, nombreuses, consacrent principalement les usages que font les jeunes d'un internet ludique et communicationnel (messageries instantanées, blogs, jeux en réseau...). Ces investigations se concentrent soit sur le contexte strictement scolaire, soit sur le contexte des pratiques culturelles ou « de loisir ». Finalement, relativement peu de travaux isolent spécifiquement l'usage d'internet par les adolescents en matière de recherche d'information. Les recherches qui ont initialement travaillé à cerner la relation des jeunes aux TICs, dès l'arrivée des " autoroutes de l'information », replaçaient largement les usages des nouveaux écrans d'alors dans le paysage des médias préexistants ${ }^{1}$. Il s'agissait de percevoir en quoi nouveaux et anciens supports de communication, interfèrent, s'annulent ou se complètent. Depuis lors, nous pouvons constater une transformation, non seulement des outils à disposition et des usages qui en sont faits, mais surtout un déplacement de la problématique elle-même. Il ne s'agit plus tant de spécifier la pratique d'internet par rapport aux autres médias d'information, que de comprendre en quoi elle est susceptible de modifier le rapport même à l'information et donc au savoir, tant elle devient exclusive. Si les pratiques internautes globales des adolescents se trouvent aujourd'hui largement dépeintes, leurs représentations et pratiques de recherche d'information sur Internet demeurent à décrire. C'est le but premier de notre travail de recherche ici présenté. En outre et s'appuyant sur les enquêtes et travaux préexistants en la matière, notre problématique s'attache à caractériser l'éventuel écart entre pratiques ordinaires, dites "informelles», et pratiques scolaires de recherche.

La pratique d'internet par les jeunes a été copieusement décrite à l'occasion d'une investigation internationale connue en France sous le nom de sa synthèse : Médiappro (Bévort et al. 2006). Elle est le premier travail du genre à embrasser les pratiques internautes réelles, quotidiennes et ordinaires des jeunes. Même si l'objet de l'étude, l'outil internet comme les acteurs, tous deux qualifiables d'« immatures », évolue constamment, ces conclusions peuvent nous être utiles comme un instantané des pratiques globales et une base sur laquelle appuyer la réflexion. Ainsi, dans les pratiques des jeunes internautes, les fonctionnalités communicationnelles arrivent en tête, avec la messagerie électronique et surtout les messageries instantanées du type «MSN Windows live messenger ». Si les pratiques d'internet des jeunes plébiscitent cet internet social et communicationnel, il est à noter que la recherche documentaire vient juste après. Le Web constitue ainsi à leurs yeux une source privilégiée d'information, le tandem Google Wikipédia remportant sans surprise les suffrages.

Les enquêtes de ce type pointent non seulement la généralisation de la pratique d'internet par les jeunes, mais surtout le fait que cette massification des pratiques s'effectue à la maison (IFOP 2005). Il faut sans doute y reconnaître l'effet du déploiement des équipements dans les foyers, en particulier ceux incluant un enfant scolarisé ${ }^{2}$. Au-delà donc de la description précieuse mais globale des pratiques des jeunes sur internet, ces travaux d'enquête installent le constat largement relayé de l'écart grandissant entre l'école et la maison. Ce fossé est constaté ou déploré, il reste à le caractériser. La question de l'écart doit en effet être très clairement posée au niveau précis des pratiques d'information. C'est ainsi une chose de percevoir que les activités de Chat et de blog ou que les jeux vidéos sont bannis des préoccupations scolaires,

\footnotetext{
${ }^{1}$ JOUET, J. PASQUIER, D. Les jeunes et l'écran. Paris : Hermès Science Publications, 1999

${ }^{2}$ BIGOT, Régis ; CROUTTE, Patricia . La diffusion des technologies de l'information dans la société française : Conditions de vie et Aspirations des Français. CREDOC, Décembre 2007
} 
tout en occupant une ample partie du temps de loisir des jeunes. Il en est une autre de se demander si la recherche d'information, deuxième grande activité des jeunes sur Internet, présente également dans les attentes scolaires, est elle-même concernée par ce hiatus. Les habitudes de recherche personnelles restent la part mal connue des pratiques numériques juvéniles. Cette méconnaissance est exacerbée du fait d'une certaine porosité des contextes d'usages, encouragée par les évolutions technologiques elles-mêmes de plus en plus individualisées et nomades. Les constats d'enquêtes font état de pratiques liées à des contextes tranchés, sphère du loisir, sphère de la vie privée, sphères du travail et de l'école, ce qui est nettement moins évident pour la recherche d'information. C'est cette porosité qui s'avère fondamentale dans la compréhension des pratiques de recherche des adolescents car elles, et les compétences qui les accompagnent ou les rendent possibles, quelle que soit leur nature, ne naissent pas isolément dans une situation étanche de pratique ordinaire ou d'apprentissage formel.

Complémentairement à cette distinction de fait établie par les enquêtes de terrain entre pratiques scolaires et formelles, et pratiques ordinaires, quotidiennes et domestiques des jeunes, il faut envisager la distinction de droit contenue en soi dans la notion d'informel (Béguin 2006). Nous pouvons définir cet informel comme ce qui se distingue d'un cadre officiel d'apprentissage, comme ce qui relève essentiellement du « temps mort » ou du loisir, $\mathrm{du}$ quotidien. A ce titre, l'informel dont il est question n'est pas absent des lieux d'apprentissage déclaré, des établissements scolaires, des cours de récréation comme des salles de classes. Notre questionnement prend donc sa source dans le constat dont font état les études et les enquêtes concernant les pratiques des jeunes sur internet à la maison et les pratiques des jeunes sur internet à l'école. Nous nous sommes fixé l'objectif de caractériser cet écart en termes de rapport à l'information, au-delà du clivage générationnel proclamé qui s'avère surtout vérifiable quant à l'assiduité et l'attrait plus forts des jeunes pour Internet et quant aux applications spécifiquement adolescentes qu'ils y développent. Ces constats d'écart ne nous renseignent pas sur le rapport à l'information entretenu via ce " média à tout faire " qu'ils privilégient aux dépens de tous les autres moyens d'information, en termes de stratégies de recherche comme de besoin d'information, et donc de rapport au savoir. A partir de notre échantillon, nous tentons de caractériser l'écart de fait comme de droit : que devient-il une fois dépassées les différences générationnelles d'assiduité et de nature de pratiques?

La tranche d'âge qui nous occupe est celle des 14-18 ans. Nous ne nous limitons pas à l'âge de la scolarité obligatoire afin d'élargir l'échantillon, tout en excluant les 11-13 ans, en début de collège, dont les pratiques sont à nos yeux bien spécifiques car intermédiaires entre les usages enfantins et les usages adolescents ${ }^{3}$. On peut observer une augmentation et surtout une diversification des pratiques dans la tranche centrale 14-17 ans. Le cap des 14-15 ans pointe le moment où la capacité à utiliser efficacement les moyens d'information et de communication devient stratégique et même décisive, pour les étapes de l'orientation scolaire et professionnelle par exemple (Delesalle 2006). Soulignons d'ailleurs que c'est à cet âge, entre la fin du collège et l'entrée au lycée, que sont le plus massivement exigés par les enseignants, exposés et autres devoirs " en autonomie », nécessitant la mise en oeuvre d'une recherche documentaire, quels que soient le niveau individuel de compétence des élèves et les ressources dont ils disposent...

A la lecture et à l'analyse des données produites à partir de nos cinquante-neuf entretiens,

\footnotetext{
${ }^{3}$ Pour autant, ceux-ci méritent l'attention de la recherche, du fait même de cette qualité du glissement comportemental qui s'opère.
} 
nous pouvons constater que les recherches à coloration scolaire occupent une place notable dans l'activité générale de recherche de ces jeunes disposant d'un poste connecté chez eux. Pour bon nombre des jeunes interrogés, c'est la somme de recherches scolaires prescrites, et de travail personnel en général, qui régule l'usage d'internet, y compris à la maison ${ }^{4}$. D'emblée, ces jeunes affirment la distinction intrinsèque qui définit recherche scolaire et recherche personnelle l'une par rapport à l'autre. Certains éléments les distinguent effectivement clairement: le lieu de la recherche, les thèmes abordés, l'attitude face aux ressources (souci de validation des contenus du côté des recherches scolaires), l'état d'esprit (envie et plaisir du côté des recherches personnelles), la trace des recherches menées ("copié/collé" du côté des recherches scolaires). D'autres aspects se répartissent plus indifféremment entre l'une et l'autre : le type de lecture et l'attention portée aux informations, le souvenir des recherches menées, la motivation, l'efficacité ressentie, le degré de précision attendu, la persévérance.

Les recherches considérées par eux comme "personnelles » se répartissent entre, d'une part, consultation de services ou de sites identifiés (type Youtube) et recherches ponctuelles de loisir qui peuvent être à peine qualifiées de « recherche » au sens propre du terme, et, d'autre part, recherches réelles, riches et intenses, souvent assez proches d'ailleurs en leurs thématiques de préoccupations d'ordre scolaire.

Ce qui ressort nettement des premières déclarations c'est bien le caractère obligatoire de la recherche à des fins scolaires. Ce caractère d'obligation marque le processus entier de la recherche d'information menée à des fins scolaires. Par « recherche scolaire » nous entendons donc un processus de recherche d'information mis en œuvre à la suite de la prescription explicite d'un enseignant et/ou portant sur un élément de cours figurant au programme de la classe concernée. La «recherche scolaire» ne se confond pas ainsi totalement avec la " recherche à l'école », elle la dépasse nettement. Si la recherche scolaire dépasse le strict contexte de l'établissement scolaire, elle ne se résume pas non plus à la pure prescription. Et si elle ne se définit pas uniquement par un contexte, la recherche scolaire se traduit au détour d'une intention. Le "besoin d'information» initial repose ici fondamentalement sur une demande, non seulement imposée mais obligatoire, et qui, potentiellement, peut s'opposer ou rejoindre les motivations propres du jeune chercheur d'information. Pour ceux, surtout, qui témoignent d'activités de recherches personnelles moindres, la définition même de la recherche d'information se confond alors avec l'idée de recherches menées pour l'école. Il faut en effet souligner que si tous les jeunes interrogés sont concernés à un moment ou à un autre par la recherche scolaire, certains d'entre eux, et pour une bonne part, ne font pas état de recherches personnelles très riches, certains déclarant même ne pas en effectuer du tout.

En outre, quelle que soit la nature de la tâche de recherche, prescrite ou librement menée, la démarche est la même pour tous. Et dans tous les cas, c'est finalement l'intérêt éprouvé vis-àvis du sujet de la recherche qui va motiver l'appropriation. Recherches «scolaires" et recherches " personnelles » sont en effet parfois difficiles à démêler et ce sont les recherches scolaires qui sont paradoxalement les moins clairement délimitées. L'écart de fait entre les deux ne suffit donc pas à cerner la réalité des pratiques de recherche informelles des adolescents. La part d'initiative personnelle, dans ces deux types de recherche, nous semble un premier levier pour tenter de les appréhender. Les jeunes interrogés qui exposent une activité de recherche personnelle réelle ont ainsi totalement intégré à celle-ci les recherches d'ordre scolaire.

\footnotetext{
${ }^{4}$ L'exploitation de nos données quantitatives fait émerger un usage d'internet à des fins de recherches scolaires devançant les recherches individuelles et le Chat/MSN lui-même.
} 
C'est le constat d'un fossé entre l'école et la maison qui est retenu, sous forme de slogan, comme l'une des conclusions les plus percutantes des investigations en matière de pratiques internautes adolescentes. Cette notion d'écart nous semble cruciale. Elle convoque Michel De Certeau et la métaphore du « braconnage », d'un usager singulier et créateur de son propre usage. L'écart entre des usages effectifs ou imaginés et des usages prévus ou prescrits est-il pour autant la seule condition d'existence d'un rôle actif de l'usager ? Rapportée au questionnement qui nous importe, cette question est cruciale car elle touche à la possibilité même de l'apprendre. Quels savoirs ou savoir-faire en matière de recherche d'information et d'accès à la connaissance les jeunes construisent-ils réellement, de par leur expérience quotidienne de l'internet? Peu de données pour l'instant nous renseignent finalement sur ce qui est réellement nouveau ou propre aux jeunes générations, si ce n'est l'attrait et la popularité de ces outils. Peu d'études associent âge et comportement d'information. Nous pouvons reprendre ici les conclusions de la récente étude menée à l'initiative de la British Library $^{5}$, qui discutent la réalité d'un comportement capable de se nourrir de lui-même, de l'expérience quotidienne de la manipulation. Au-delà d'une « autodidaxie générationnelle » supposée et de "manières de faire » comme petits arrangements avec la technique, il faut poser la question de l'appropriation d'internet par les jeunes en termes de rapport au savoir.

BEGUIN, A. Pourquoi faut-il étudier les pratiques informelles des apprenants en matière d'information et de documentation ? Colloque international: Savoirs et acteurs de la formation, Rouen, 18-19-20 mai 2006

BEVORT, Evelyne; BRÉDA, Isabelle (synthèse réalisée pour la France). Mediappro : Appropriation des nouveaux médias par les jeunes : une enquête européenne en éducation aux médias. Paris: Centre de Liaison de l'Enseignement et des Médias d'Information (CLEMI), 2006

DELESALLE, Cécile ; GOVINDASSAMY, Sophie ; NIAMBOSSOU, Reine. Les pratiques et usages des jeunes en matière d'information : synthèse du rapport. Institut National de la Jeunesse et de l'Education Populaire ; Vérès consultants, décembre 2006

DONNAT, Olivier ; LEVY, Florence. Approche générationnelle des pratiques culturelles et médiatiques. DEPS : Culture prospective, 2007

Institut Français d'Opinion Publique (IFOP) pour la Délégation Interministérielle à la Famille du Ministère du Travail, des Relations sociales et de la Solidarité. Les usages d'Internet par les adolescents : deuxième vague. Septembre 2005

MESSIN, Audrey. De l'usage d'Internet à la «culture de l'écran ». Journées doctorales GDR TIC et Société, Avril 2005.

PIETTE, Jacques ; Pons, Christian-Marie ; Giroux, Luc. Les jeunes et Internet 2006 :

Appropriation des nouvelles technologies. Rapport final de l'enquête. QUEBEC, Ministère de la Culture et des Communications : Mars 2007

RIGAUD, Claude. Les adolescents. La lettre d'information, n¹9, INRP, juin 2006

\footnotetext{
${ }^{5}$ University College London (UCL) CIBER group. Information behaviour of the researcher of the future. London: University College, CIBER Briefing paper. Janvier 2008.
} 